\title{
Crossing Number of Toroidal Graphs ${ }^{\star}$
}

\author{
János Pach ${ }^{1}$ and Géza Tóth ${ }^{2}$ \\ 1 City College, CUNY and Courant Institute of Mathematical Sciences, \\ New York University, New York, NY, USA \\ pach@cims.nyu.edu \\ 2 Rényi Institute, Hungarian Academy of Sciences, Budapest, Hungary \\ geza@renyi.hu
}

\begin{abstract}
It is shown that if a graph of $n$ vertices can be drawn on the torus without edge crossings and the maximum degree of its vertices is at most $d$, then its planar crossing number cannot exceed $c d n$, where $c$ is a constant. This bound, conjectured by Brass, cannot be improved, apart from the value of the constant. We strengthen and generalize this result to the case when the graph has a crossing-free drawing on an orientable surface of higher genus and there is no restriction on the degrees of the vertices.
\end{abstract}

\section{Introduction}

Let $S_{g}$ be the compact orientable surface with no boundary, of genus $g$. Given a simple graph $G$, a drawing of $G$ on $S_{g}$ is a representation of $G$ such that the vertices of $G$ are represented by points of $S_{g}$ and the edges are represented by simple (i.e., non-selfintersecting) continuous arcs in $S_{g}$, connecting the corresponding point pairs and not passing through any other vertex. The crossing number of $G$ on $S_{g}, \mathrm{cr}_{g}(G)$, is defined as the minimum number of edge crossings over all drawings of $G$ in $S_{g}$. For $\operatorname{cr}_{0}(G)$, the "usual" planar crossing number, we simply write $\operatorname{cr}(G)$.

Let $G$ be a graph of $n$ vertices and $e$ edges, and suppose that it can be drawn on the torus without crossing, that is, $G$ satisfies $\operatorname{cr}_{1}(G)=0$. How large can $\operatorname{cr}(G)$ be? Clearly, we have $\operatorname{cr}(G)<\left(\begin{array}{l}e \\ 2\end{array}\right)$, and this order of magnitude can be attained, as shown by the following example. Take five vertices and connect any pair of them by $\frac{e}{20}$ vertex-disjoint paths of lengths two. In any drawing of this graph in the plane, every subdivision of $K_{5}$ gives rise to a crossing. Therefore, the number of crossings must be at least $\frac{e^{2}}{400}$.

Peter Brass suggested that this estimate can be substantially improved if we impose an upper bound on the degree of the vertices. More precisely, we have

Theorem 1. Let $G$ be a graph of $n$ vertices with maximum degree $d$, and suppose that $G$ has a crossing-free drawing on the torus. Then we have $\operatorname{cr}(G) \leq c d n$, where $c$ is a constant.

\footnotetext{
* János Pach has been supported by NSF Grant CCR-00-98246, and by grants from PSC-CUNY, OTKA, NSA, and BSF. Géza Tóth has been supported by OTKA-T038397 and T-046246.
} 
For $d \geq 3$, the bound in Theorem 1 cannot be improved, apart from the value of the constant $c$. Consider the following example. Let $d \geq 4, G=C_{k} \times C_{k}$, where $k=\sqrt{n / d}$ is a large integer and $C_{k}$ denotes a cycle of length $k$. Obviously, this graph can be drawn on the torus without crossings. On the other hand, by a result of Salazar and Ugalde [SU04], its planar crossing number is larger than $\left(\frac{4}{5}-\varepsilon\right) k^{2}$, for any $\varepsilon>0$, provided that $k$ is large enough. Substitute every edge $e$ of $G$ by $\left\lfloor\frac{d}{4}\right\rfloor$ new vertices, each connected to both endpoints of $e$. The resulting graph $G^{\prime}$ has at most $n$ vertices, each of degree at most $d$. It can be drawn on the torus with no crossing, and its planar crossing number is at least

$$
\left(\frac{4}{5}-\varepsilon\right) k^{2} \times\left\lfloor\frac{d}{4}\right\rfloor^{2}>\frac{1}{100} n d .
$$

To see this, it is enough to observe that there is an optimal drawing of $G^{\prime}$ in the plane with the property that any two paths of length two connecting the same pair of vertices cross precisely the same edges. The same construction can be slightly modified to show that $\operatorname{cr}(G)$ can also grow linearly in $n$ if the maximum degree $d$ is equal to three.

Theorem 1 can be generalized as follows.

Theorem 2. Let $G$ be a graph of $n$ vertices of maximum degree $d$ that has a crossing-free drawing on $S_{g}$, the orientable surface of genus $g$. Then we have $\operatorname{cr}(G) \leq c_{d, g} n$, where $c_{d, g}$ is a constant depending on $d$ and $g$.

We can drop the condition on the maximum degree and obtain an even more general statement.

Theorem 3. Let $G$ be a graph of $n$ vertices with degrees $d_{1}, d_{2}, \ldots, d_{n}$, and suppose that $G$ has a crossing-free drawing on $S_{g}$. Then we have

$$
\operatorname{cr}(G) \leq c_{g} \sum_{i=1}^{n} d_{i}^{2},
$$

where $c_{g}$ is a constant depending on $g$.

To simplify the presentation and to emphasize the main idea of the proof, in Section 2 first we settle the simplest (planar) case (Theorem 1). In Section 3, we reduce Theorem 3 to a similar upper bound on the crossing number of $G$ in $S_{g-1}$ (Theorem 3.1). This latter result is established in Section 4.

\section{The Simplest Case: Proof of Theorem 1}

We can assume that $d \geq 3$. It is sufficient to prove that $\operatorname{cr}(G) \leq \operatorname{cd}(n-1)$ holds for any two-connected graph $G$ satisfying the conditions. Indeed, if $G$ is disconnected or has a cut vertex, then it can be obtained as the union of two graphs $G_{1}$ and $G_{2}$ with $n_{1}$ and $n_{2}$ vertices that have at most one vertex in common, so that 
we have $n_{1}+n_{2}=n$ or $n+1$. Arguing for $G_{1}$ and $G_{2}$ separately, we obtain by induction that

$$
\operatorname{cr}(G)=\operatorname{cr}\left(G_{1}\right)+\operatorname{cr}\left(G_{2}\right) \leq c d\left(n_{1}-1\right)+c d\left(n_{2}-1\right) \leq c d(n-1),
$$

as required.

Let $G$ be a two-connected graph with maximum degree $d$ and $\operatorname{cr}_{1}(G)=0$. Fix a crossing-free drawing of $G$ on the torus. We can assume that the boundary of each face is connected. Indeed, if one of the faces contains a cycle not contractible within the face, then cutting the torus along this cycle we do not damage any edge of $G$. Therefore, $G$ is a planar graph and there is nothing to prove.

If our drawing is not a triangulation, then by adding $O(n)$ extra vertices and edges we can turn it into one so that the maximum degree of the vertices increases by at most a factor of three. We have to apply the following easy observation.

Lemma 2.1. Let $G$ be a two-connected graph with $n$ vertices of degree at most $d(d \geq 3)$. Suppose that $G$ has a crossing-free drawing on the orientable surface of genus $g$ such that the boundary of each face is connected. Any such drawing can be extended to a triangulation of the surface with at most $19 n+36(g-1)$ vertices of maximum degree at most $3 d$.

Proof. First consider a cycle $f=x_{1} x_{2} \ldots x_{n(f)}$ bounding a single face in the drawing of $G$. Note that some vertices $x_{i} \in V(G)$ and even some edges may appear along this cycle several times. Take a simple closed curve $\gamma_{0}=p_{1} p_{2} \ldots p_{n(f)}$ inside the face, running very close to $f$ and passing through the (new) points $p_{i}$ in this cyclic order. In the ring between $f$ and $\gamma_{0}$, connect each vertex $x_{i}$ to $p_{i}$ and $p_{i+1}$ (where $\left.p_{n(f)+1}:=p_{1}\right)$.

Divide $\gamma_{0}$ into $m_{0}:=\left\lceil\frac{n(f)}{d-1}\right\rceil$ connected pieces, each consisting of at most $d$ vertices, such that the last vertex of each piece $\pi_{i}$ is the first vertex of $\pi_{i+1}$, where $1 \leq i \leq m_{0}$ and $\pi_{m_{0}+1}:=\pi_{1}$. Place a simple closed curve $\gamma_{1}=q_{1} q_{2} \ldots q_{m_{0}}$ in the interior of $\gamma_{0}$. In the ring between $\gamma_{0}$ and $\gamma_{1}$, connect each $q_{i}$ to all points in $\pi_{i}$. (If $m_{0}=1$ or 2 , then $\gamma_{1}$ degenerates into a point or a single edge.) If $\gamma_{1}$ has more than three vertices, repeat the same procedure for $\gamma_{1}$ in the place of $\gamma_{0}$, and continue as long as the interior of the face is not completely triangulated. We added

$$
n(f)+m_{0}+m_{1}+\ldots<n(f)+n(f)+\frac{n(f)}{2}+\frac{n(f)}{4}+\ldots<3 n(f)
$$

new vertices, and their maximum degree is at most $d+4$. The degree of every original vertex of $f$ increased by at most twice the number of times it appeared in $f$.

If we triangulate every face of $G$ in the above manner, the resulting drawing $G^{\prime}$ defines a triangulation of the surface with fewer than $n+\sum_{f} 3 n(f) \leq n+$ $6|E(G)|$ vertices, each of degree at most $d^{\prime}:=3 d$. By Euler's formula, we have $n+6|E(G)| \leq n+18(n-2+2 g)$, as required.

In the sequel, slightly abusing the notation, we write $G$ for the triangulation $G^{\prime}$ and $d$ for its maximum degree $d^{\prime}$. 
If $G$ has no noncontractible cycle, i.e., no cycle represented on the torus by a closed curve not contractible to a point, then we are done, because $G$ is a planar drawing so that $\operatorname{cr}(G)=0$. Otherwise, choose a noncontractible cycle $C$ with the minimum number of vertices, fix an orientation of $C$, and let $k:=|V(C)|$. Let $E_{l}$ (and $E_{r}$ ) denote the set of edges not belonging to $C$ that are incident to at least one vertex of $C$ and in a small neighborhood of this vertex lie on the left-hand side (respectively right-hand side) of $C$. Note that the sets $E_{l}$ and $E_{r}$ are disjoint, but this fact is not necessary for the proof.

Replace $C$ by two copies, $C_{r}$ and $C_{l}$, lying on its right-hand side and left-hand side. Connect each edge of $E_{r}$ (respectively $E_{l}$ ) to the corresponding vertex of $C_{r}$ (respectively $C_{l}$ ). Cut the torus along $C$, and attach a disk to each side of the cut.

The resulting spherical (planar) drawing $G_{1}$ represents a graph, slightly different from $G$. To transform it into a drawing of $G$, we have to remove $C_{l}$ and (re)connect the edges of $E_{l}$ to the corresponding vertices of $C_{r}$. In what follows, we describe how to do this without creating too many crossings.

Let $\hat{G}_{1}$ denote the dual graph of $G_{1}$, that is, place a vertex of $\hat{G}_{1}$ in each face of $G_{1}$, and for any $e \in E\left(G_{1}\right)$ connect the two vertices assigned to the faces meeting at $e$ by an edge $\hat{e} \in E\left(\hat{G}_{1}\right)$. Let $r$ and $l$ denote the vertices of $\hat{G}_{1}$ lying in the faces bounded by $C_{r}$ and $C_{l}$.

Lemma 2.2. In $\hat{G}_{1}$, there are $k$ vertex-disjoint paths between the vertices $r$ and $l$.

Proof. By Menger's theorem, the maximum number $p$ of (internally) vertexdisjoint paths connecting $r$ and $l$ in $\hat{G}_{1}$ is equal to the minimum number of vertices whose deletion separates $r$ from $l$. Choose $p$ such separating vertices, and denote the corresponding triangular faces of $G$ by $f_{1}, \ldots, f_{p}$. The interior of the union of these faces must contain a noncontractible closed curve that does not pass through any vertex of $G$. Let $\delta$ be such a curve whose number of intersection points with the edges of $G$ is minimum. Choose an orientation of $\delta$. Let $e_{1}, \ldots, e_{q}$ denote the circular sequence of edges of $G$ intersected by $\delta$. By the minimality of $\delta$, we have $q \leq p$, because the interior of each triangle $f_{i}$ contains at most one maximal connected piece of $\delta$. Let $v_{i}$ be the right endpoint of $e_{i}$ with respect to the orientation of $\delta$. Notice that $v_{i}$ is adjacent to or identical with $v_{i+1}$, for every $1 \leq i \leq q$ (where $v_{q+1}:=v_{1}$ ). Therefore, the circular sequence of vertices $v_{1}, \ldots, v_{q}$ induces a cycle in $G$ that can be continuously deformed to $\delta$. Thus, we have a noncontractible cycle of length $q \leq p$ in $G$, which implies that $k$, the length of the shortest such cycle, is at most $p$, as required.

By Lemma 2.1, the graph $\hat{G}$ has at most $2|V(G)| \leq 38 n$ vertices. According to Lemma 2.2, there is a path connecting $r$ and $l$ in $\hat{G}$ with fewer than $\frac{38 n}{k}$ internal vertices. The corresponding faces of $G_{1}$ form a "corridor" $B$ between $C_{r}$ and $C_{l}$. Delete now the vertices of $C_{l}$ from $G_{1}$. Pull every edge in $E_{l}$ through $B$, and connect each of them to the corresponding vertex of $C_{r}$. See Figures 1 and 2 . Notice that during this procedure one can avoid creating any crossing between edges belonging to $E_{l}$.

We give an upper bound on the number of crossings in the resulting planar drawing of $G$. Using that $|C|=k$ and $\left|E_{l}\right| \leq d k$, we can conclude that by 

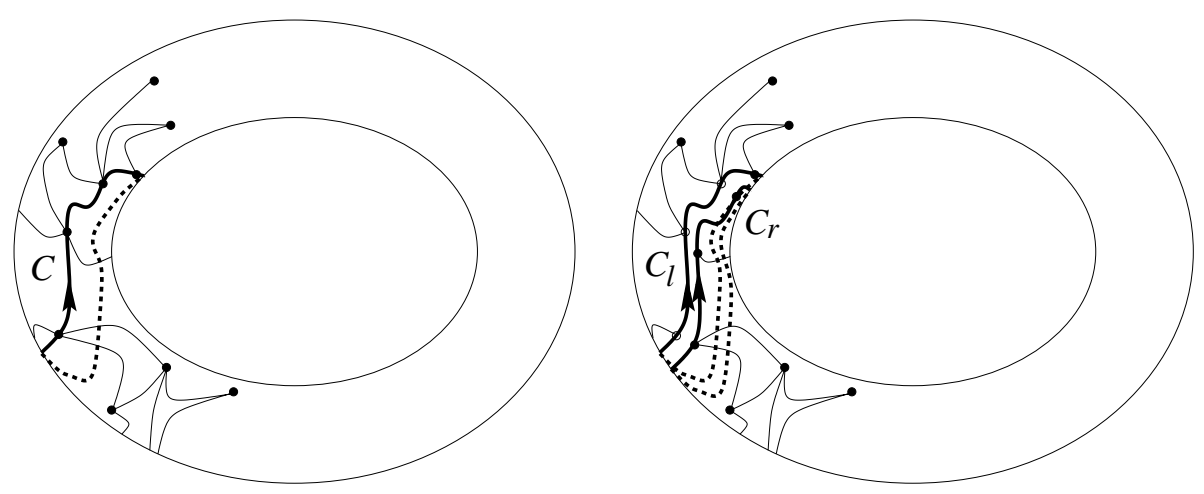

Fig. 1. $C$ is the shortest noncontractible cycle
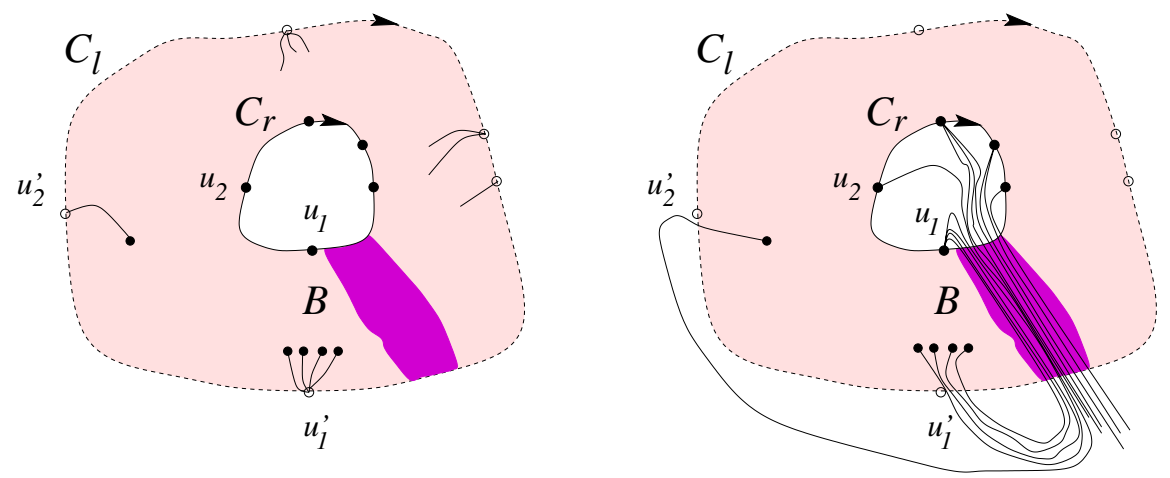

Fig. 2. Pulling the edges in $E_{l}$ through the corridor $B$

pulling each edge through the corridor $B$, we create at most $\frac{38 n}{k}$ crossings per edge. Thus, the total number of crossings cannot exceed $d k \cdot \frac{38 n}{k}=38 d n$, which completes the proof of Theorem 1 .

\section{Reducing Theorem 3 to Theorem 3.1}

Given a graph $G$, let $n(G)$ and $\sigma(G)$ denote the number of vertices of $G$ and the sum of the squares of their degrees.

Theorem 3 provides an upper bound for the crossing number of a graph $G$ that can be drawn on $S_{g}$ without crossing. Next we show that this bound can be deduced by repeated application of the following result. In each step, we reduce the genus of the surface by one.

Theorem 3.1. Let $G$ be a two-connected graph with $\operatorname{cr}_{g}(G)=0$. Then we have $\mathrm{cr}_{g-1}(G) \leq c_{g}^{*} \sigma(G)$, for some constant $c_{g}^{*} \geq 1$. 
Proof of Theorem 3 using Theorem 3.1. As in the proof of Theorem 1, we can assume that $G$ is two-connected. Consider a crossing-free drawing of $G_{0}:=G$ on $S_{g}$. According to Theorem 3.1, $G_{0}$ can be drawn on $S_{g-1}$ with at most $c \sigma(G)$ crossings. Place a new vertex at each crossing, and apply Theorem 3.1 to the resulting graph $G_{1}$. Proceeding like this, we obtain a series of graphs $G_{2}, G_{3}, \ldots, G_{g}$, drawn on $S_{g-2}, S_{g-3}, \ldots, S_{0}$, respectively, with no crossing.

We claim that for any $i, 0 \leq i \leq g$,

$$
\sigma\left(G_{i}\right) \leq(17)^{i}\left(\prod_{g-i<j \leq g} c_{j}^{*}\right) \sigma(G)
$$

holds. This is obviously true for $i=0$. Let $0<i \leq g$, and assume that the claim has already been verified for $i-1$. Notice that, apart from the original vertices of $G_{i-1}$, every other vertex of $G_{i}$ has degree four. Thus, applying Theorem 3.1 to the graph $G_{i-1}$ that had a crossing-free drawing on $S_{g-i+1}$, we obtain

$$
\begin{gathered}
\sigma\left(G_{i}\right) \leq \sigma\left(G_{i-1}\right)+16 \mathrm{cr}_{g-i}\left(G_{i-1}\right) \leq \sigma\left(G_{i-1}\right)+16 c_{g-i+1}^{*} \sigma\left(G_{i-1}\right) \\
\leq\left(1+16 c_{g-i+1}^{*}\right)(17)^{i-1}\left(\prod_{g-i+1<j \leq g} c_{j}^{*}\right) \sigma(G) \leq(17)^{i}\left(\prod_{g-i<j \leq g} c_{j}^{*}\right) \sigma(G),
\end{gathered}
$$

which proves the claim.

It follows from the construction that $G_{g}$ is a planar graph, and we have

$$
n\left(G_{g}\right)-n(G)<\sigma\left(G_{g}\right) \leq 17^{g}\left(\prod_{j=1}^{g} c_{j}^{*}\right) \sigma(G) .
$$

Replacing the $n\left(G_{g}\right)-n(G)$ "new" vertices of $G_{g}$ by proper crossings, we obtain a drawing of $G$ in the plane with at most $17^{g}\left(\prod_{j=1}^{g} c_{j}^{*}\right) \sigma(G)$ crossings. This completes the proof of Theorem 3 .

\section{Reducing the Genus by One: Proof of Theorem 3.1}

It remains to prove Theorem 3.1.

All noncrossing closed curves $C$ on $S_{g}$ belong to one of the following three categories:

1. $C$ is contractible (to a point);

2. $C$ is noncontractible and twosided, i.e., it separates $S_{g}$ into two connected components;

3. $C$ is noncontractible and onesided.

Let us cut the surface $S_{g}$ along $C$, and attach a disk along each side of the cut. If $C$ is contractible, we obtain two surfaces: one homeomorphic to $S_{g}$ and 
the other homeomorphic to the sphere $S_{0}$. If $C$ is noncontractible and twosided, then we obtain two surfaces homeomorphic to $S_{a}$ and $S_{b}$, for some $a, b>0$ with $a+b=g$. Finally, if $C$ is noncontractible and onesided, then we get only one surface, $S_{g-1}$ [MT01].

First we need an auxiliary statement, interesting on its own right.

Theorem 4.1. Let $G$ be a graph with a crossing-free drawing on $S_{g}$. If $G$ has no noncontractible onesided cycle, then $G$ is a planar graph.

Proof. We follow the approach of Cairns and Nikolayevsky CN00, developed to handle a similar problem on generalized thrackles. Let $S$ be a very small closed neighborhood of the union of all edges of the drawing of $G$ on $S_{g}$. Then $S$ is a compact connected surface whose boundary consists of a finite number of closed curves. Attaching a disk to each of these closed curves, we obtain a surface $S^{\prime}$ with no boundary. We show that $S^{\prime}$ is a sphere. To verify this claim, consider two closed curves, $\alpha^{\prime}$ and $\beta^{\prime}$, on $S^{\prime}$. They can be continuously deformed into closed walks, $\alpha_{1}$ and $\beta_{1}$, along the edges of $G$. Let $\alpha$ and $\beta$ be the corresponding closed walks along the edges of $G$ in the original drawing on $S_{g}$. By the assumption, $\alpha$ divides $S_{g}$ into two parts, therefore, $\beta$ crosses $\alpha$ an even number of times. Since the original drawing of $G$ on $S_{g}$ was crossing-free, every crossing between $\alpha$ and $\beta$ occurs at a vertex of $G$. Using the fact that in the new drawing of $G$ on $S^{\prime}$, the cyclic order of the edges incident to a vertex is the same as the cyclic order of the corresponding edges in the original drawing, we can conclude that $\alpha_{1}$ and $\beta_{1}$ cross an even number of times. It is not hard to argue that then the same was true for $\alpha^{\prime}$ and $\beta^{\prime}$. Thus, $S^{\prime}$ is a surface with no boundary in which any two closed curves cross an even number of times. This implies that $S^{\prime}$ is a sphere. Consequently, we have a crossing-free drawing of $G$ on the sphere, that is, $G$ is a planar graph.

Proof of Theorem 3.1. As in the previous section, let $\sigma(G)$ denote the the sum of the squared degrees of the vertices of $G$. A grid of size $k \times k$ is the cross product $P_{k} \times P_{k}$ of two paths of length $k$. The vertices of $P_{k} \times P_{k}$ with degrees less than four are said to form the boundary of the grid. The proof of Theorem 3.1 is based on the same idea as that of Theorem 1, but some important details have to be modified.

Suppose that $G$ is a two-connected graph of $n$ vertices, drawn on $S_{g}$ without crossing. We can also assume that $G$ has no crossing-free drawing on $S_{g-1}$, otherwise Theorem 3.1 is trivially true. In particular, it follows that every face of the drawing of $G$ on $S_{g}$ has a connected boundary.

Replace each vertex $v$ of degree $d(v)>4$ by a grid of size $d(v) \times d(v)$ and connect the edges incident to $v$ to distinct vertices on the boundary of the grid, preserving their cyclic order. The resulting crossing-free drawing of $G^{\prime}$ has at most $\sigma(G)$ vertices, each of degree at most four. Every face has a connected boundary, so that we can apply Lemma 2.1 to turn $G^{\prime}$ into a triangulation $G^{\prime \prime}$ with at most $19 \sigma(G)+36(g-1)$ vertices, each of degree at most twelve. Restricting $G^{\prime}$ and $G^{\prime \prime}$ to any grid substituting for a vertex in $G$, the only difference between them is that each quadrilateral face in $G^{\prime}$ is subdivided by 
one of its diagonals into two triangles in $G^{\prime \prime}$. Color all edges along the boundaries of the grids blue, and all other grid and diagonal edges of $G^{\prime \prime}$ that lie in the interior of some grid red.

If $G^{\prime \prime}$ has no noncontractible onesided cycle, then we are done by Theorem 4.1. Otherwise, pick such a cycle $C$ with the smallest number $k$ of vertices. Without increasing its length too much, we can replace all red edges of $C$ by blue edges. Indeed, the first vertex and the last vertex of any maximal red path in $C$ must belong to the boundary of the same grid. Replace each such path by the shortest blue path connecting its first and last vertices along the boundary of the grid containing them. The resulting cycle $C^{\prime}$ is noncontractible, onesided, and its length is at most $2 k$. It has no red edges, and we can assume without loss of generality that it does not intersect itself. Fix an orientation of $C^{\prime}$.

Let $E_{l}$ (and $E_{r}$ ) denote the set of edges not belonging to $C^{\prime}$ that are incident to at least one vertex of $C^{\prime}$ and in a small neighborhood of this vertex lie on the left-hand side (respectively right-hand side) of $C^{\prime}$.

Replace $C^{\prime}$ by two copies, $C_{r}$ and $C_{l}$, lying on its right-hand side and lefthand side. Connect each edge of $E_{r}$ and $E_{l}$ ) to the corresponding vertex of $C_{r}$ and $C_{l}$. Cut $S_{g}$ along $C$, and attach a disk to each side of the cut. The resulting surface is $S_{g-1}$, and it contains a crossing-free drawing $G_{1}$ of a graph slightly different from $G^{\prime \prime}$. To obtain a drawing of $G^{\prime \prime}$ from $G_{1}$, we have to remove $C_{l}$ and (re)connect the edges of $E_{l}$ to the corresponding vertices of $C_{r}$ without creating too many crossings.

Let $\hat{G}_{1}$ be the dual drawing of $G_{1}$ on $S_{g-1}$. Let $r$ (respectively $l$ ) be the vertex of $\hat{G}_{1}$ lying in the face bounded by $C_{r}$ (respectively $C_{l}$ ). Color blue each vertex of $\hat{G}_{1}$ that corresponds to a face lying inside a grid in $G^{\prime \prime}$.

Repeating the proof of Lemma 2.2, we obtain

Lemma 4.2. In $\hat{G}_{1}$, there are $k$ vertex-disjoint paths between the vertices $r$ and $l$.

The number of cells in $G_{1}$ is equal to the number of cells in $G^{\prime \prime}$ plus 2 . Therefore, by Euler's formula, $\hat{G}_{1}$ has at most

$2\left|V\left(G^{\prime \prime}\right)\right|+4(g-1)+2 \leq 2(19 \sigma(G)+36(g-1))+4(g-1)+2<40(\sigma(G)+2 g)$

vertices. Thus, by Lemma 4.2, there is a path $P(r l)$ between $r$ and $l$, of length at most $40(\sigma(G)+2 g) / k$. Replacing all blue vertices of $P(r l)$ by others, we obtain a new path $P^{\prime}(r l)$, not much longer than $P(r l)$. First observe that $r$ and $l$, the two endpoints of $P(r l)$, are not blue. Let $u v_{1} v_{2} \ldots v_{j} v$ be an interval along $P$ such that all $v_{i}$ 's are blue $(1 \leq i \leq j)$, but $u$ and $v$ are not. Then the faces corresponding to $u$ and $v$ must be adjacent to the boundary of some grid in $G_{1}$. These two faces are connected by two chains of faces following the outer boundary of the grid. Replace $v_{1}, v_{2}, \ldots, v_{j}$ by the sequence of vertices corresponding to the shorter of these two chains. Since the degree of every vertex in $G_{1}$ is at most twelve, the length of this chain is at most $12 j$. Repeating this procedure for each maximal blue interval of $P(r l)$, we obtain a new path $P^{\prime}(r l)$, whose length is at most $480(\sigma(G)+2 g) / k$. 
The corresponding faces of $G_{1}$ form a "corridor" $B$ between $C_{r}$ and $C_{l}$. Now delete $r, l$, and the vertices of $C_{l}$. In the same way as in the proof of Theorem 1 , "pull" all edges of $E_{l}$ through $B$, and connect them to the corresponding vertices of $C_{r}$. This step can be carried out without creating any crossing between the edges in $E_{l}$.

Now we count the number of crossings in the resulting drawing. Since $\left|C^{\prime}\right| \leq$ $2 k,\left|E_{l}\right| \leq 20 k$. Pulling them through the corridor $B$, we create no more than $480(\sigma(G)+2 g) / k$ crossings per edge, that is, at most $X:=9600(\sigma(G)+2 g)$ crossings altogether.

Deleting the extra vertices and edges from $G_{1}$ and collapsing each grid into a vertex, we obtain a drawing of $G$ on $S_{g-1}$, in which the number of crossings cannot exceed $X$. This concludes the proof of Theorem 3.1.

Acknowledgement. We are very grateful to Zoltán Szabó (Princeton) for many valuable suggestions.

\section{References}

[CN00] G. Cairns and Y. Nikolayevsky, Bounds for generalized thrackles, Discrete Comput. Geom. 23 (2000), 191-206.

[MT01] B. Mohar and C. Thomassen: Graphs on surfaces, Johns Hopkins Studies in the Mathematical Sciences. Johns Hopkins University Press, Baltimore, MD, 2001.

[SU04] G. Salazar and E. Ugalde: An improved bound for the crossing number of $C_{m} \times C_{n}$ : a self-contained proof using mostly combinatorial arguments, Graphs Combin. 20 (2004), 247-253. 